\title{
Bibliographie de la Société de Notre-Dame de Montréal (1639-1663), accompagnée de notes critiques et historiques (suite)
}

\section{Marie-Claire Daveluy}

Volume 8, numéro 3, décembre 1954

URI : https://id.erudit.org/iderudit/301674ar

DOI : https://doi.org/10.7202/301674ar

Aller au sommaire du numéro

Éditeur(s)

Institut d'histoire de l'Amérique française

ISSN

0035-2357 (imprimé)

1492-1383 (numérique)

Découvrir la revue

Citer ce document

Daveluy, M.-C. (1954). Bibliographie de la Société de Notre-Dame de Montréal (1639-1663), accompagnée de notes critiques et historiques (suite). Revue d'histoire de l'Amérique française, 8(3), 449-454.

https://doi.org/10.7202/301674ar 


\title{
BIBLIOGRAPHIE *
}

Bibliographie de la Société de Notre-Deme de Montréal (1639-1663), accompagnée de notes critiques et historiques.

\section{DEUXIEMEE PARTIE}

\author{
Bio-Bibliographie des Associés de Montréal \\ (suite)
}

\section{6. - JEANNE MANCE, 1606-1673. - Bibliographie (suite). \\ 2. - Note sur les ouvrages des contemporains de Jeanne Mance.}

Le nom de Jeanne Mance apparaît pour la première fois dans un écrit destiné au grand public de France, le 9 may 1641. Il s'agit d'un entrefilet, dans la Gazette de Renaudot, relatant son départ pour la Nouvelle-France et qui la présente comme un ascète "ne se nourrissant que de pain et d'eau". (Voir le No 6 de notre bibliographie dans le volume V, no 1 de la RHAF (juin 1951), p. 143). Nous y avons décrit minutieusement la source de nos renseignements sur le départ de Jeanne et de Maisonneuve, accompagnés de colons-fondateurs, en 1641.

Les Relations des Jésuites, pour les années 1640-1641,1642, 1643 et $1651^{48}$, mentionnent l'arrivée de Jeanne Mance, à Québec (mais sans préciser le quantième), les premiers baptêmes des Sauvages avec Jeanne Mance et Maisonneuve comme marraine et parrain, la fondation de l'hôpital (automne 1642, au Fort de Ville-Marie) et aussi d'autres détails concernant les gestes d'apôtre de Jeanne.

146. - [Premier Registre d'état civil à Ville-Marie 1642].

Conservé aux Archives de la Fabrique de l'église Notre-Dame,

*Voir Revue d'bistoire de l'Amérique française, V, no $1: 139-147$; 296 307; 3: 445-460; 4 : 603-616; VI, no $1: 146-150 ; 2$ : 297-305; $3: 458-463 ; 4$ : 595-605; VII : no 3 : 457-461; 4 : 586-592; VIII, no 2 : 292-306.

48. Les éditions originales des Relations pour les années 1640-1641, 1642, ont été décrites dans notre bibliographie, au volume $\mathrm{V}$, no 1 de cette Revue, p. 143 et 144 , nos 7 et 8 . 
à Montréal. Baptêmes, mariages et sépultures.

N.B. Le Premier Registre de Ville-Marie, tel que nous pouvons le constater aujourd'hui, ne possède pas d'actes originaux pour les années allant de 1642 à 1646 . Rédigées tout probablement sur des feuilles volantes par les missionnaires de l'époque, elles furent copiées par le Père Claude Pijart, lorsqu'il prit charge de la mission en 1646. C'est, en tout cas, l'explication la plus plausible à présenter, puisque de 1642 à 1646, c'est la même main (celle du Père Claude Pijart) qui consigne les actes au Registre, quoique les cérémonies eussent été accomplies par des missionnaires différents. Les copies se trouvent, du reste, authentiquées par le desservant de 1646. De 1647 à 1672 (en ce qui concerne la participation de Jeanne Mance en qualité de marraine ou de témoin) tous les actes sont des pièces originales. Notons seulement que Jeanne Mance, du 20 septembre 1641 au 19 septembre 1672, fut 83 fois marraine, soit de Français, soit de Sauvages. Les actes du temps des missionnaires Jésuites (de 1642 à 1657) sont tous rédigés en latin.

147. - 1643. - Relation / de ce qui s'est passé / en la Nouvelle-France, / ès année 1643, Envoyée au R. Père Provincial de la / Compagnie de Jésus, de la Province de France / Par le P. Barthélemy Vimont de la mesme / Compagnie, Supérieur de la Résidence de Kébec. / [Ornement typographique : la marque de l'Imprimerie]. / A Paris / chez Sebastien Cramoisy, imprimeur ordinaire / du Roy, ruë S. Jacques, aux Cigognes. / M.D.C.XLII. / Avec privilège du Roy. / 17 x $11 \mathrm{~cm}$. - Reliure d'époque en parchemin.

N.B. L'édition originale que nous avons consultée appartient à la Bibliothèque Municipale de Montréal (Salle Philéas Gagnon, Collection Witton). Rédigée par le Père Vimont. D'intéressants détails sont fournis sur la vie à Ville-Marie, de l'automne 1642 au printemps 1643, notamment sur la fondation, au Fort, à l'automne de 1642, de l'bôpital dirigé par Jeanne Mance, et commandité par Madame Claude de Bullion.

148. - 1650-1651. - Relation / de ce qui s'est passé / de plus remarquable / ès Missions des Pères de la / Compagnie de Jésus, / en la / Nouvelle-France / ès années 1650 \& 1651. / Envoyée au R.P. Provincial de la Province de France / Par le Père Paul Ragueneau, supérieur des / Missions de la mesme Compagnie /. A Paris, / Sebastien Cramoisy, l'imprimeur ordinaire du / Roy \& de la Reyne, / et Gabriel Cramoisy / rue S. Jacques, aux Cigognes. / M.D.C. LII. / Avec privilège du Roy. / [2] - 146 - [1] pages. $17 \times 11 \mathrm{~cm}$. Reliure d'époque en parchemin. 
N.B. Nous avons consulté l'édition originale conservée à la Bibliothèque Municipale de Montréal. Le texte louant la charité apostolique de Jeanne Mance se trouve dans le chapitre intitulé : De la résidence de Montréal (p. 40-42).

Puis viennent les Véritables Motifs ..., 1643 (Voir le No 14 de notre bibliographie dans le vol. V, no 1 de la RHAF (juin 1951), p. 147-148). Jeanne Mance y est considérée comme une "grande servante de Dieu".

Le Journal des Jésuites, de 1645 à 1668, imprimé à Québec en 1871. (Voir le no 22 de notre bibliographie dans le vol. V, no 2 de la RHAF (septembre 1951), p. 299-300). Nous y trouvons fidèlement notés les trois voyages de Jeanne Mance en France, 1649-1650, 1658-1659, 1662-1664.

Un Règlement concernant l'administration de l'bostel-Dieu de St-Joseph en ladite isle de Montréal, 14 avril 1648. Cette mesure disciplinaire illustre la probité scrupuleuse de la cofondatrice de l'Hôtel-Dieu. L'hôpital de Montréal conserve un précieux manuscrit de Jeanne Mance, administratrice de l'hôpital. Il est intitulé : Mémoire des choses que les Messieurs de Montréal ont donné et fourny à l'bospital, de 1644 à 1660. (Voir le no 31 de notre bibliographie dans le vol. V, no 2 (Septembre 1951), p. 307).

1663 (9 mars). Il importe de citer ici, touchant l'administration de l'Hôtel-Dieu par Jeanne Mance, le Contract de donation de l'Isle de Montréal au Séminaire de Saint-Sulpice, par les Associés de Montréal. (Voir le no 43 de notre Bibliographie dans le vol. 5, no 3 de cette Revue (décembre 1951). L'infirmière de Ville-Marie dont on blâmait la décision extrême prise en 1653, pour sauver Ville-Marie agonisante, c'est-à-dire affectant à la levée d'une recrue, certains capitaux appartenant à l'hôpital et donnés par Mme de Bullion, les Messieurs de Montréal, dans l'Acte de Donation du 9 mars 1663, l'exonèrent parfaitement en cette occasion comme en toutes les autres. L'éloge de l'administratrice couvre les courageuses résolutions adoptées de 1642 à 1663.

1672-1673. Histoire du Montréal, 1640-1672, par Dollier de Casson, p.s.s., imprimée pour la première fois à Montréal en 1868, accompagnée de notes et de pièces justificatives recueillies par Jacques Viger. Nous nous sommes servis cependant de la $3 e$ éd. préparée par Ralph Flenley (Toronto 1928), texte anglais et français en regard. (Voir le No 46 de notre Bibliographie dans le vol. 5, no 3, de cette Revue (décembre 1951). Nous ne saurions trop souligner l'importance d'une telle source fondamentale concernant Jeanne Mance. M. Dollier connut et interrogea souvent l'infirmière vieillissante. Il servait à Ville-Marie en 1666, il occupait le poste de supérieur de Saint-Sulpice à la mort de l'héroïne (18 juin 1673). Son appréciation de la vocation de l'admirable femme est magnifique, et s'accompagne de détails historiques assez sûrs, mais qu'il faut tout de même contrôler puisque 
M. Dollier recueillait les souvenirs de témoins relatant des événements survenus trente ans plus tôt. D'ailleurs, l'honnête historien nous en prévient et s'en excuse. Il admet les déficiences d'un travail modeste comme le sien; il avoue n'avoir ni lu, ni vu aucun récit antérieur à celui qu'il offre en toute simplicité d'accent à ses confrères sulpiciens infirmes ou malades. Seule, la tradition orale subsistait donc à Montréal, à l'époque de M. Dollier. Les faits glorieux accomplis par les colons-fondateurs ${ }^{49}$, de 1642 à 1666, restent d'un témoin "ex auditu a videntibus". Au contraire, à partir de 1666, c'est en témoin oculaire que $M$. Dollier tient la plume.

1673. - Le Mémoire de M. de Maisonneuve sur les Affaires de Montréal, adressé à $M$. Louis Tronson, le $3 e$ supérieur de Saint-Sulpice (à partir de 1676) et daté de 1673, est indispensable à consulter sur la malheureuse question des 22,000 livres qui vint assombrir les dernières années de Jeanne Mance tout comme celles de $M$. de Maisonneuve. On trouve dans le Mémoire de celui-ci une justification de la conduite du gouverneur et de l'infirmière de Ville-Marie, lors des années critiques de 1651 à 1653. (Voir la description du document au No 47 de cette bibliographie (vol. V, no 3, décembre 1951).

1697. - Les Mémoires de Soeur Morin de l'Hôtel-Dieu de Montréal ont une importance égale comme source narrative fondamentale sur l'infirmière de Ville-Marie. Nous savons que Marie Morin, née en 1649, à Québec, fille de Noël Morin et d'Hélène Desportes, entrait à l'Hôtel-Dieu de Ville-Marie en 1662. Elle vécut durant onze ans auprès de Jeanne Mance; elle l'entendit souvent raconter les souvenirs de Langres, de Paris, et des jours de la fondation de Montréal. L'annaliste s'exprime avec une candeur charmante sur ce dont elle a été témoin, sans trop se préoccuper de la précision des dates. Elle ne se livre pas à un travail d'historien, certes, la bonne hospitalière, mais raconte de son mieux, dans leurs grandes lignes, les événements qui se sont gravés dans sa sérieuse petite tête. Elle aussi, comme M. Dollier, n'écrit pas pour le grand public. Elle s'adresse aux Hospitalières de la Flèche, en France, parce que celles-ci l'en ont prié. M. Faillon cite 127 fois Soeur Morin dans sa biographie de Jeanne Mance. C'est dire que cet auteur s'appuie dans la majeure partie de son oeuvre, sur M. Dollier de Casson, sulpicien, et Soeur Morin, de l'Hôtel-Dieu de Montréal. Le manuscrit de cette dernière est conservé aux Archives de notre Hôtel-Dieu. C'est un petit in-folio en papier où court la large et haute écriture de la moniale et dont l'orthographe se révèle fort capricieuse. . . comme tant d'autres écrivains du XVIIe siècle, pourtant si bien disants. L'Hôtel-Dieu possède maintenant une copie photostatée de l'original, empêchant ainsi les manipulations imprudentes ou trop fréquentes. (Pour la description des Annales de Soeur Morin éditées par MM. Fauteux, Massi-

49. M. Faillon appréciait l'oeuvre narrative de $M$. Dollier. Il en fait une des sources fondamentales de la Vie de Jeanne Mance qu'il publiait en 1854. Il cite 109 fois M. Dollier dans son ouvrage. 
cotte et Bertrand, voir le No 52 de notre Bibliograpbie, dans le vol. V, no 3 de cette Revue (décembre 1951), p. 157).

Voici maintenant à leur ordre chronologique les Ecrits autographes de la Bienbeureuse Marguerite Bourgeoys, cette amie fidèle de Jeanne Mance depuis son arrivée à Ville-Marie en 1653 , et qui recueillait son dernier soupir, le dimanche soir, 18 juin 1673. Son témoignage est infiniment précieux quant au portrait moral et spirituel de l'infirmière qui ressort des nombreux récits de la première et sainte éducatrice de Montréal. Evidemment, ce sont ici encore des Mémoires, et la Bienheureuse les racontait aux heures de sa vieillesse, en 1698. Mais tout en nous souciant de contrôler les dates et plusieurs détails des Ecrits, nous ne pouvons que faire grand cas d'un incomparable témoin oculaire. Voici la description que fait Dom Jamet, O.S.B., d'une copie authentique du manuscrit original (aujourd'hui hélas disparu). "Quant aux Ecrits autographes, déclare cet érudit dans sa $V$ ie de Marguerite Bourgeoys (Montréal, 1942, 2 vol., voir pour la citation, vol. I, introduction, p. 7), [ils ont été] disséminés aux quatre coins du Canada français, après 1818 (date de la publication de la Vie de Soeur Bourgeoys attribuée à M. Etienne Montgolfier, p.s.s.). M. Faillon, [cependant en 1852] a pu en faire récupérer les fragments. De ces pièces tronquées, on a composé une mosaïque plus ou moins exacte, où la patience et la critique aidant, l'historien actuel peut encore se reconnaître. Ce recueil original a disparu dans l'incendie de la Congrégation en 1893. Une copie littérale, heureusement, en avait été prise peu auparavant. Les recoupements faits dans les citations de [l'abbé] Glandelet [du Séminaire de Québec] et dans celle de la Vie de 1818 permettraient d'affirmer que nous avons encore la majeure partie des Mémoires de Marguerite Bourgeoys..." Le témoignage du religieux bénédictin (décédé ces toutes dernières années) est d'autant plus précieux qu'il est le dernier érudit ayant consulté avec un jugement critique alerte, cette source indispensable de l'histoire de Montréal et de ses colons-fondateurs, toujours à l'état de manuscrit. Aucune édition critique, en effet, n'a été publiée de cette oeuvre jusqu'à ce jour.

Nous ajouterons enfin, une troisième mémorialiste à celles qui précèdent : Mère Françoise Juchereau de Saint-Ignace, née en 1650, à la Seigneurie de Beauport, aux environs de Québec, entrée au Cloître de l'HôtelDieu de Québec, à l'âge de douze ans, et s'y éteignant en 1723, à l'âge de 73 ans. L'autorité et la distinction de cette petite-fille de Robert Giffard,

50. Jeanne Mance décédait à l'Hôtel-Dieu, le dimanche soir, 18 juin 1673. C'est d'après l'Inventaire de ses biens dressé dès le lendemain, le 19 juin, que nous connaissons la date exacte de sa mort. Ce précieux document est conservé aux Archives judiciaires de Montréal, tout comme l'acte de sépulture de Jeanne Mance se trouve dans les Registres des baptêmes, mariages et sépultures de la paroisse de Montréal. . . pour l'année 1673. L'acte de dépost du coeur de deffunte Damoiselle Jeanne Mance est conservé dans le minutier Basset, aux Archives judiciaires de Montréal, et porte aussi la date du 19 juin 1673. 
devinrent bientôt telles qu'elle fut élue sept fois supérieure de l'Hôtel-Dieu de l'année 1683 à l'année 1717. C'est Mère Juchereau qui se prononça la première sur l'ensemble de la vie bienfaisante de Jeanne Mance. "Elle est décédée en odeur de sainteté, note-t-elle au lendemain de sa mort. Elle fut enterrée honorablement dans l'église des Hospitalières de Saint-Joseph, à Montréal." 50 (Pour la description du manuscrit original commencée vers 1699, comme pour l'édition critique luxueuse qu'en publiait Dom Albert Jamet, O.S.B., en 1639, voir le No 53 de notre bibliographie (Vol. V, no 3, de la RHAF (décembre 1951), p. 457-458).

\section{3. - Note sur quelques écrits modernes.}

Le sulpicien-historien, Etienne-Michel Faillon, occupe une place prépondérante dans la littérature historique canadienne, au milieu du XIXe siècle. Dès 1841, avec la biographie de M. Olier (voir le No 107 de cette bibliographie, RHAF, VI, no 4 (mars 1953), p. 599), où il est question des commencements de Montréal, et dont la publication coïncidait avec le deuxième centenaire de la fondation de notre ville. A cette époque même M. Faillon portait des regards attentifs sur l'histoire du Canada. Il débutait par la vie de personnages aussi saints qu'audacieux dans leurs initiatives de grande envergure. Ce furent ses travaux de base. Ils l'acheminèrent vers son Histoire de la colonie française (voir le No 54 de cette bibliographie, RHAF, vol. V, no 3 (décembre 1951), p. 459) qu'il ne conduisit qu'à l'année 1675, malgré la publication de trois volumes de format in-quarto. C'était en marquer toute la richesse des sources. On reproche à $\mathrm{M}$. Faillon d'avoir fait à Montréal la part du lion et de se montrer très partial envers les Jésuites. Nous ne pouvons nier la justesse de ces critiques, mais nous nous gardons avant tout d'ignorer les découvertes de ce merveilleux érudit. Le Canada du XVIIe siècle en ressort illuminé dans combien de ses détails embués jusque là. Cependant, comme nous retrouverons à quelques pages près, dans notre bibliographie, le consciencieux biographe de Jeanne Mance, nous nous contenterons des quelques observations ci-dessus.

149. - 1861 - Translation des corps des Soeurs défuntes de l'Hôtel-Dieu de Montréal. Nécrologie des Religieuses hospitalières de St-Joseph, décédées depuis l'établissement de ce monastère à Montréal ou Ville-Marie, en l'année 1659 jusqu'en 1860. Inhumées dans l'église. Montréal, des presses à vapeur de Plinguet \& Cie, 26, rue St-Gabriel, 1861. 14 pages. In-8.

N,B. Cette pièce rarissime est souvent attribuée à l'auteur de l'Annuaire de Ville-Marie (Montréal, 1864), le notaire Huguet-Latour, de la Société

51. Rappelons qu'au moment où $\mathrm{M}$. Souart s'occupait de l'Acte de dépost du coeur de Jeanne Mance, l'inhumation de son corps, d'après les termes de ce document, venait d'avoir lieu dans l'église, seule église paroissiale de l'époque (1673). Mère Juchereau de Saint-Ignace, de son côté, nous apprend dans ses Mémoires (1699), que Jeanne Mance "fut enterrée honorablement dans l'église". 
historique de Montréal (fondée en 1857). Elle tire son importance du fait que l'on y affirme de nouveau ${ }^{51}$ la présence encore possible de quelques ossements de Jeanne Mance, enterrée dans l'église de l'Hôtel-Dieu en 1673.

Nous notons maintenant parmi les écrivains d'aujourd'hui les ouvrages (sauf les biographies que nous allons étudier séparément) de: Georges Goyau (voir le No 86 de notre bibliographie, RHAF, vol. VI, no 1, (juin 1952), p. 148-149), de Mère Mondoux, r.h. (voir le No 100 de notre bibliographie, RHAF, vol. VI, no 2 (septembre 1952), p. 304), de Camille Bertrand, archiviste, (voir idem, No 101, p. 304-305).

Mentionnons encore le Procès-verbal de l'exhumation des restes mortels des religieuses hospitalières de Saint-Joseph de l'Hôtel-Dieu de Montréal, décédées à l'ancien Hôtel-Dieu de la rue Saint-Paul, en vue d'établir la reconnaissance du corps de Jeanne Mance que l'on croit possible d'y retrouver. Cette pièce manuscrite est conservée à l'Hôtel-Dieu de Montréal et datée de 1947. Le résultat des recherches, hélas, s'avéra nul.

Enfin, nous possédons sur Jeanne Mance et la vénération des Canadiens à son endroit deux précieux mémoires de Mère Mondoux, l'archiviste distinguée de l'Hôtel-Dieu de Montréal. Le premier mémoire, dont nous avons parlé dans le numéro précédent de cette Revue, est à l'état de manuscrit et date de 1643. Voici le second qui a été publié l'an dernier.

150. - 1953. - Les Hautes Vertus de Jeanne Mance. Sa réputation de sainteté par Soeur Mondoux, r.h. Montréal, le Comité des Fondateurs, 8100, boulevard Saint-Laurent, 1953.

15 pages. In-8. (Collection, "Textes", no 7).

$$
\text { (à suivre) }
$$

Marie-Claire Daveluy 